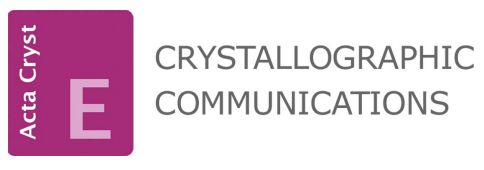

ISSN 2056-9890

Received 20 October 2021

Accepted 5 November 2021

Edited by H. Ishida, Okayama University, Japan

Keywords: crystal structure; intramolecular $\mathrm{N}-$ $\mathrm{H}$... O interactions; $\mathrm{C}-\mathrm{H} \cdots \pi$ interactions; Hirshfeld surface analysis.

CCDC reference: 1983650

Supporting information: this article has supporting information at journals.iucr.org/e

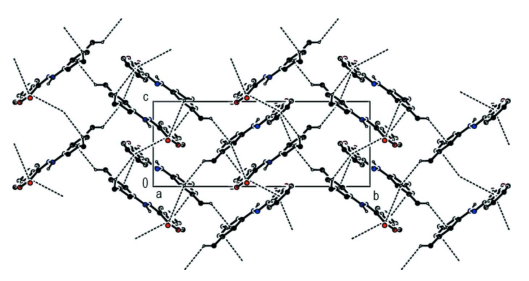

\section{Crystal structure and Hirshfeld surface analysis of 3-[2-(3,5-dimethylphenyl)hydrazinylidene]benzo- furan-2(3H)-one}

\author{
Zeliha Atioğlu, ${ }^{\text {a }}$ Mehmet Akkurt, ${ }^{\mathrm{b}}$ Namiq Q. Shikhaliyev, ${ }^{\mathrm{c}}$ Ulviyya F. Askerova, ${ }^{\mathrm{c}}$ \\ Sevinc H. Mukhtarova, ${ }^{c}$ Rizvan K. Askerov ${ }^{\mathrm{c}}$ and Ajaya Bhattarai ${ }^{\mathrm{d} *}$
}

\begin{abstract}
a Department of Aircraft Electrics and Electronics, School of Applied Sciences, Cappadocia University, Mustafapaşa, 50420 Ürgüp, Nevşehir, Turkey, 'b Department of Physics, Faculty of Sciences, Erciyes University, 38039 Kayseri, Turkey, 'Organic Chemistry Department, Baku State University, Z. Xalilov str. 23, Az, 1148 Baku, Azerbaijan, and d Department of Chemistry, M.M.A.M.C (Tribhuvan University) Biratnagar, Nepal. *Correspondence e-mail: bkajaya@yahoo.com
\end{abstract}

In the title compound, $\mathrm{C}_{16} \mathrm{H}_{14} \mathrm{~N}_{2} \mathrm{O}_{2}$, the 2,3-dihydro-1-benzofuran ring system is essentially planar and makes a dihedral angle of $3.69(7)^{\circ}$ with the dimethylphenyl ring. The molecular conformation is stabilized by an intramolecular $\mathrm{N}-$ $\mathrm{H}$... O hydrogen bond with an $S(6)$ ring motif. In the crystal, molecules are connected by $\mathrm{C}-\mathrm{H} \cdots \pi$ and $\pi-\pi$ stacking interactions, forming a layer lying parallel to the $(11 \overline{1})$ plane. One methyl group is disordered over two orientations, with occupancies of 0.67 (4) and 0.33 (4). Hirshfeld surface analysis indicates that the most important contributions to the crystal packing are from $\mathrm{H} \cdots \mathrm{H}(51.2 \%), \mathrm{O} \cdots \mathrm{H} / \mathrm{H} \cdots \mathrm{O}(17.9 \%), \mathrm{C} \cdots \mathrm{H} / \mathrm{H} \cdots \mathrm{C}(15.2 \%)$ and $\mathrm{C} \cdots \mathrm{C}$ $(8.1 \%)$ contacts.

\section{Chemical context}

Hydrazones have many applications in diverse areas, such as in optical data storage, as molecular switches and antimicrobial agents, in non-linear optics, molecular recognition, dye-sensitized solar cells, color-changing materials, catalysis, liquid crystals, etc., mainly because of the azo-to-hydrazo tautomerism/isomerism and the optical properties of $-\mathrm{N}=\mathrm{N}-$ unit (Maharramov et al., 2018; Ma et al., 2020, 2021; Viswanathan et al., 2019). Not only $E / Z$ isomerization, but also azohydrazone tautomerism is important in organic and the coordination chemistry of hydrazone dyes (Ma et al., 2017a,b; Mahmoudi et al., 2017, 2018). The design of hydrazone dyes with electron donor or acceptor substituents has led to multidentante ligands, the corresponding coordination compounds of which have been applied effectively as catalysts in oxidation and $\mathrm{C}-\mathrm{C}$ coupling reactions (Mahmudov et al., 2013; Mizar et al., 2012). Moreover, the functional properties of hydrazones or their metal complexes can be regulated by attaching functional groups to the $=\mathrm{N}-\mathrm{NH}-$ unit (Gurbanov et al., 2020a,b; Kopylovich et al., 2011; Mahmudov et al., 2020; Shixaliyev et al., 2014). Thus, we have attached $\mathrm{C}=\mathrm{O}$ groups and furan and aryl rings to the $=\mathrm{N}-\mathrm{NH}-$ moiety, leading to a new hydrazone compound, $(Z)-3-[2-(3,5-$ dimethylphenyl)hydrazinylidene]benzofuran-2(3H)-one, which can form intermolecular interactions. 
<smiles>Cc1cc(C)cc(N/N=C2\C(=O)Oc3ccccc32)c1</smiles>

\section{Structural commentary}

The molecular conformation of the title compound is stabilized by an intramolecular $\mathrm{N}-\mathrm{H} \cdots \mathrm{O}$ hydrogen bond ( $\mathrm{N} 2-$ $\mathrm{H} 2 \cdots \mathrm{O} 2$; Table 1) with an $S(6)$ ring motif (Fig. 1). The 2,3dihydro-1-benzofuran ring system $(\mathrm{O} 1 / \mathrm{C} 1-\mathrm{C} 8)$ of the title compound is essentially planar [maximum deviations = -0.031 (2) $\AA$ for C3 and 0.026 (2) $\AA$ for C6] and makes a dihedral angle of $3.69(7)^{\circ}$ with the dimethylphenyl C9-C14 ring. In the molecule, the aromatic $\mathrm{C} 9-\mathrm{C} 14$ ring and the $\mathrm{C}=\mathrm{N}-\mathrm{NH}-$ unit are almost coplanar with a dihedral angle of $4.8(8)^{\circ}$ between them.

\section{Supramolecular features}

In the crystal, molecules are connected by $\mathrm{C}-\mathrm{H} \cdots \pi$ interactions $\quad\left[\mathrm{C} 15-\mathrm{H} 15 A \cdots C g 3^{\mathrm{i}}\right.$ and $\mathrm{C} 16-\mathrm{H} 16 F \cdots C g 2^{\mathrm{ii}}$; symmetry codes as given in Table 1 ; Fig. 2] and $\pi-\pi$ stacking interactions $\left[C g 1 \cdots C g 2^{\text {iii }}=3.6227(11) \AA\right.$, slippage $=1.226 \AA$; $C g 1 \cdots C g 3^{\text {ii }}=3.7128(10) \AA$, slippage $=1.339 \AA$; Symmetry

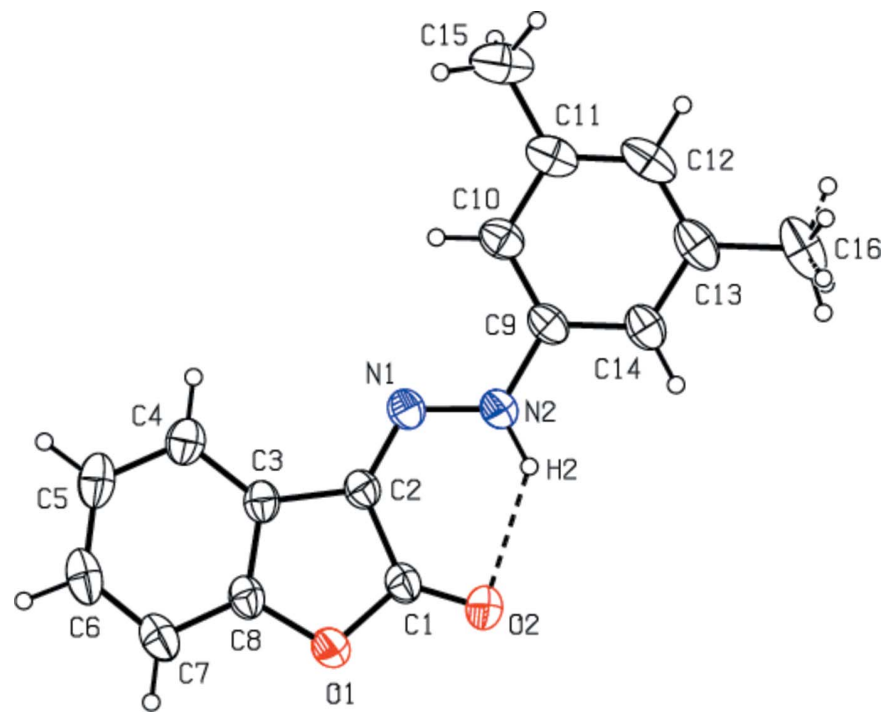

Figure 1

The molecular structure of the title compound with displacement ellipsoids for the non-hydrogen atoms drawn at the $30 \%$ probability level.
Table 1

Hydrogen-bond geometry $\left(\AA,^{\circ}\right)$.

$C g 2$ and $C g 3$ are the centroids of the $\mathrm{C} 3-\mathrm{C} 8$ and $\mathrm{C} 9-\mathrm{C} 14$ rings, respectively.

\begin{tabular}{lllll}
\hline$D-\mathrm{H} \cdots A$ & $D-\mathrm{H}$ & $\mathrm{H} \cdots A$ & $D \cdots A$ & $D-\mathrm{H} \cdots A$ \\
\hline $\mathrm{N} 2-\mathrm{H} 2 \cdots \mathrm{O} 2$ & $0.93(2)$ & $2.12(2)$ & $2.840(2)$ & $133.8(16)$ \\
$\mathrm{C} 15-\mathrm{H} 15 A \cdots C g 3^{\mathrm{i}}$ & 0.96 & 2.90 & $3.591(3)$ & 130 \\
$\mathrm{C} 16-\mathrm{H} 16 F \cdots C g 2^{\mathrm{ii}}$ & 0.96 & 2.92 & $3.715(3)$ & 141 \\
\hline
\end{tabular}

Symmetry codes: (i) $x,-y-\frac{1}{2}, z-\frac{3}{2}$; (ii) $-x+1,-y+1,-z+1$.

codes: (ii) $-x+1,-y+1,-z+1$; (iii) $-x+2,-y+1,-z+2]$, where $C g 1$ and $C g 2$ are the centroids of the oxolane O1/C1$\mathrm{C} 3 / \mathrm{C} 8$ and benzene $\mathrm{C} 3-\mathrm{C} 8$ rings, respectively, of the 2,3-di-

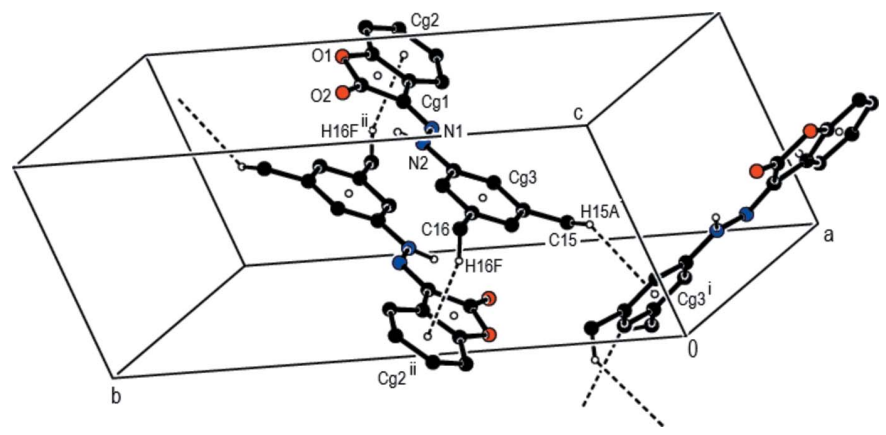

Figure 2

A partial packing view of the title compound, showing the $\mathrm{C}-\mathrm{H} \cdots \pi$ interactions (dashed lines). Only $\mathrm{H}$ atoms involved in the interactions and $\mathrm{N}$-bound $\mathrm{H}$ atoms are shown for clarity [Symmetry codes: (i) $x,-y-\frac{1}{2}$, $z-\frac{3}{2}$; (ii) $-x+1,-y+1,-z+1$.]

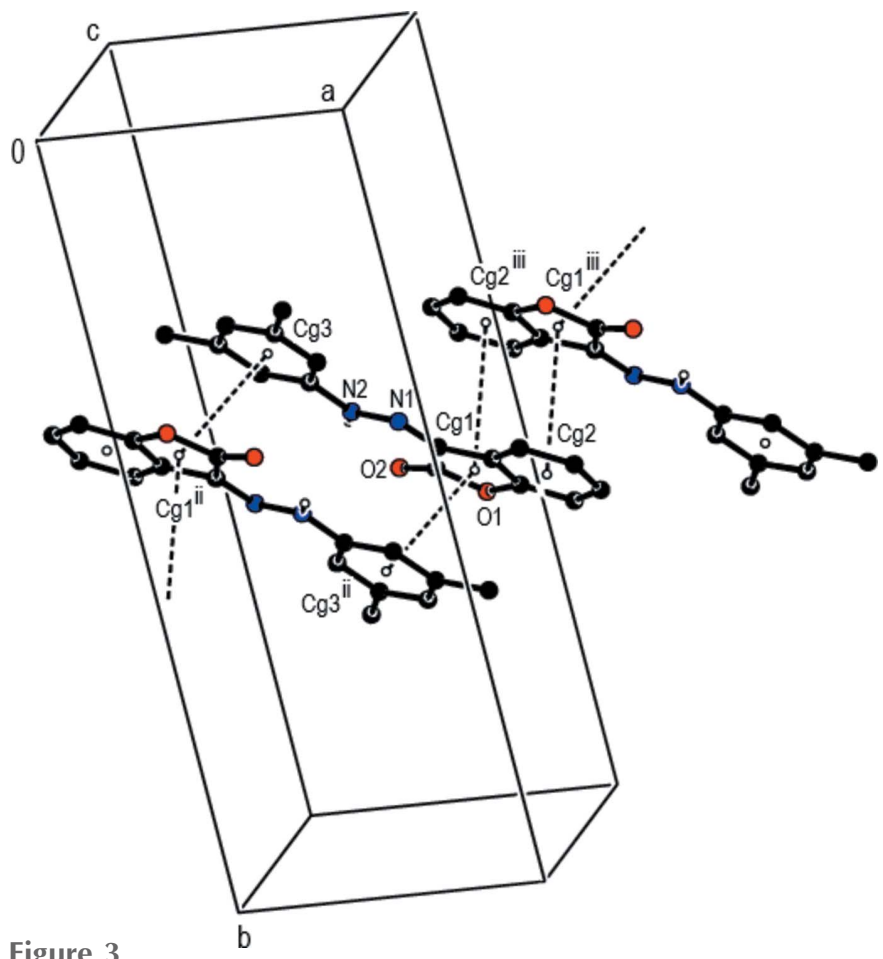

Figure 3

A partial packing view of the title compound, showing the $\pi-\pi$ stacking interactions (dashed lines). Only $\mathrm{N}$-bound $\mathrm{H}$ atoms are shown for clarity [Symmetry codes: (ii) $-x+1,-y+1,-z+1$; (iii) $2-x, 1-y, 2-z$.] 


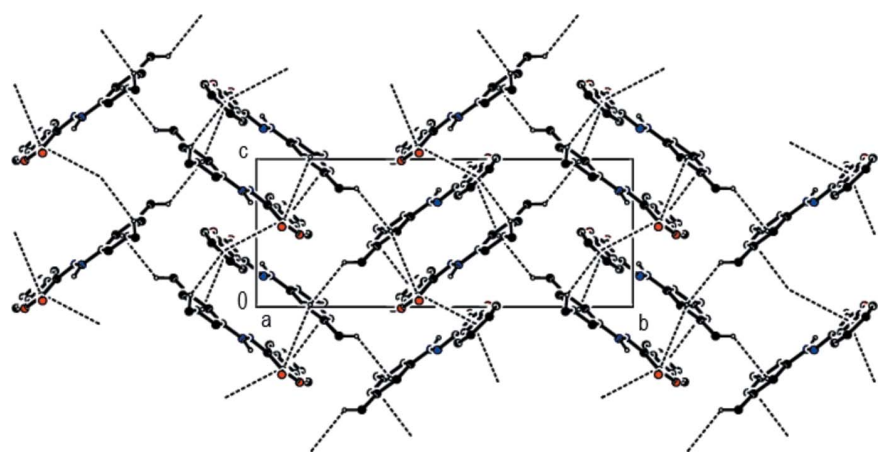

Figure 4

A packing diagram of the title compound viewed along the $a$-axis, showing $\mathrm{C}-\mathrm{H} \cdots \pi$ interactions (dashed lines). Only $\mathrm{H}$ atoms involved in the interactions and $\mathrm{N}$-bound $\mathrm{H}$ atoms are shown for clarity.

hydro-1-benzofuran ring system while $C g 3$ is the centroid of the dimethylphenyl C9-C14 ring (Fig. 3). These interactions link the molecules into a layer structure lying parallel to the (111̄) plane (Fig. 4).

\section{Hirshfeld surface analysis}

Crystal Explorer17 (Turner et al., 2017) was used to perform a Hirshfeld surface analysis and generate the associated twodimensional fingerprint plots, with a standard resolution of the three-dimensional $d_{\text {norm }}$ surfaces plotted over a fixed color scale of -0.0001 (red) to 1.5993 (blue) a.u. (Fig. 5a). All of the disordered $\mathrm{H}$ atoms of the $\mathrm{C} 16$ methyl group were taken into account together. The shape-index of the Hirshfeld surface is a tool to visualize the $\pi-\pi$ stacking by the presence of adjacent red and blue triangles; if there are no adjacent red and/or blue triangles, then there are no $\pi-\pi$ interactions. Fig. $5 b$ clearly indicates that there are $\pi-\pi$ interactions in the title compound.

Two-dimensional fingerprint plots for the $\mathrm{H} \cdots \mathrm{H}, \mathrm{O} \cdots \mathrm{H} /$ $\mathrm{H} \cdots \mathrm{O}, \mathrm{C} \cdots \mathrm{H} / \mathrm{H} \cdots \mathrm{C}$ and $\mathrm{C} \cdots \mathrm{C}$ contacts are presented in Fig. 6. $\mathrm{H} \cdots \mathrm{H}$ interactions, which are located in the middle region of the fingerprint plot, contribute the most to overall crystal packing, with $51.2 \%$ (Fig. $6 b$ ). The $\mathrm{O} \cdots \mathrm{H} / \mathrm{H} \cdots \mathrm{O}$ contacts contribute $17.9 \%$ (Fig. $6 c$ ) to the Hirshfeld surface, while the $\mathrm{C} \cdots \mathrm{H} / \mathrm{H} \cdots \mathrm{C}$ contacts contribute $15.2 \%$ (Fig. $6 d$ ), resulting in a pair of distinctive wings. The $\mathrm{C} \cdots \mathrm{C}$ interactions account for $8.1 \%$ of the Hirshfeld surface. The percentage

Table 2

Percentage contributions of interatomic contacts to the Hirshfeld surface of the title compound.

\begin{tabular}{ll}
\hline Contact & Percentage contribution \\
\hline $\mathrm{H} \cdots \mathrm{H}$ & 51.2 \\
$\mathrm{O} \cdots \mathrm{H} / \mathrm{H} \cdots \mathrm{O}$ & 17.9 \\
$\mathrm{C} \cdots \mathrm{H} / \mathrm{H} \cdots \mathrm{C}$ & 15.2 \\
$\mathrm{C} \cdots \mathrm{C}$ & 8.1 \\
$\mathrm{~N} \cdots \mathrm{C} / \mathrm{C} \cdots \mathrm{N}$ & 4.2 \\
$\mathrm{~N} \cdots \mathrm{H} / \mathrm{H} \cdots \mathrm{N}$ & 1.9 \\
$\mathrm{O} \cdots \mathrm{C} / \mathrm{C} \cdots \mathrm{O}$ & 0.9 \\
$\mathrm{~N} \cdots \mathrm{N}$ & 0.6 \\
$\mathrm{O} \cdots \mathrm{O}$ & 0.1 \\
\hline
\end{tabular}
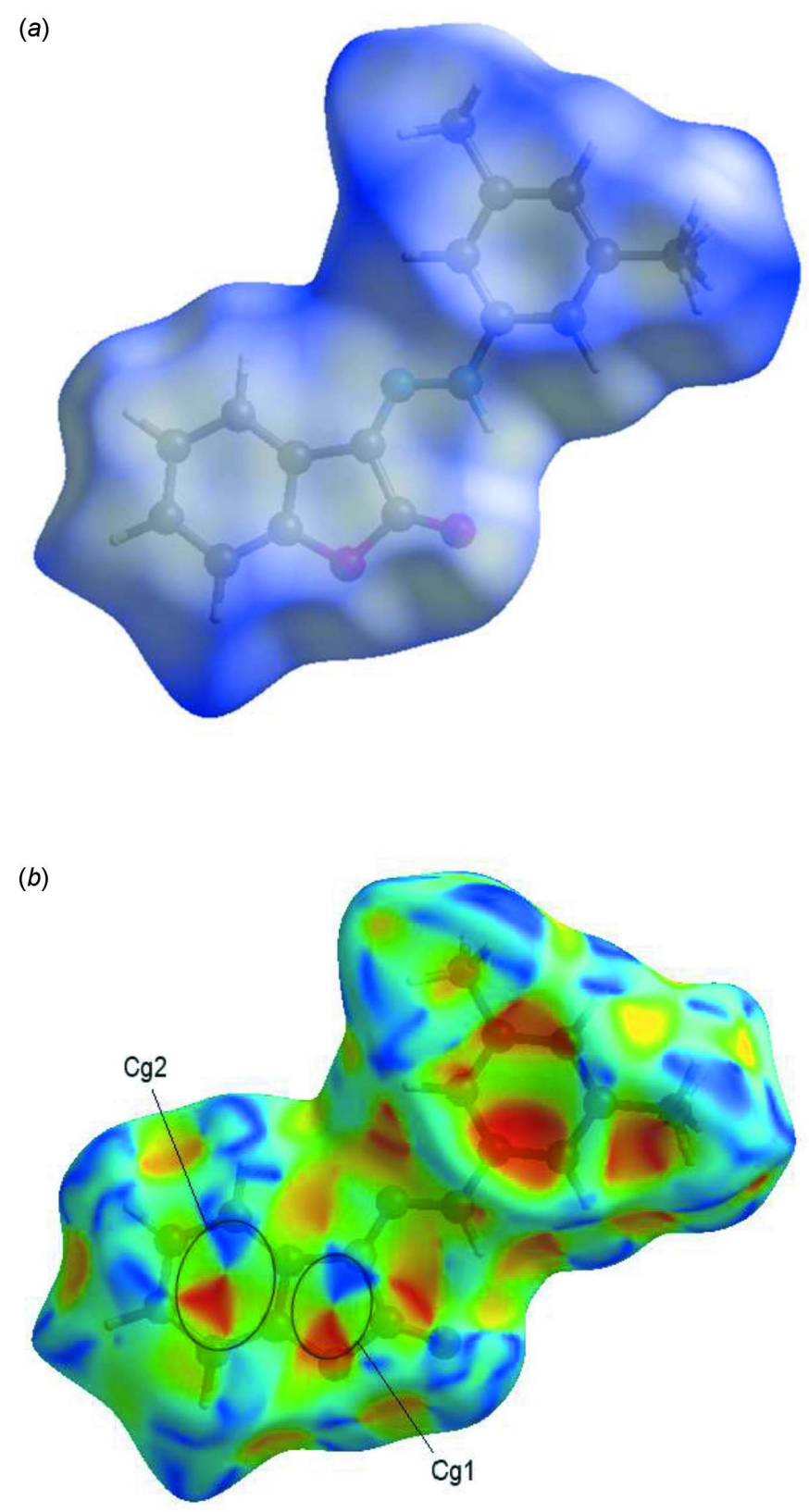

Figure 5

Hirshfeld surfaces of the title molecule, $(a)$ mapped with $d_{\text {norm }}$ in the range -0.0001 to 1.5993 a.u. and $(b)$ plotted over shape-index.

contributions to the Hirshfeld surface including other minor ones are summarized in Table 2.

\section{Database survey}

A search of the Cambridge Crystallographic Database (CSD version 5.42, updated September 2021; Groom et al., 2016) for the 1-benzofuran-2(3H)-one unit gave 220 hits. Of these, the compound most similar to the title compound is 7-methoxy-3(2-phenylhydrazinylidene)-1-benzofuran-2(3H)-one, I (CSD refcode IBADIC; Atioğlu et al., 2021). Four compounds reported by Oliveira et al. (2019) are closely related to the title compound, viz. 2-(4-nitro-1H-imidazol-1-yl)- $N^{\prime}$-[1-(pyridin-2- 


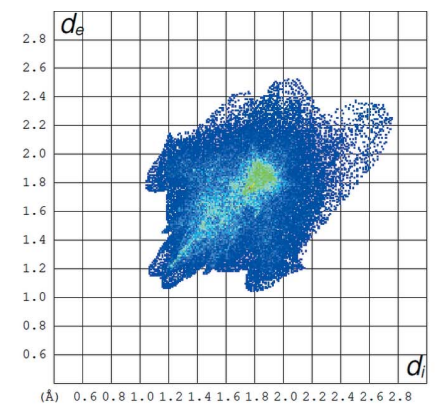

(a) All...All

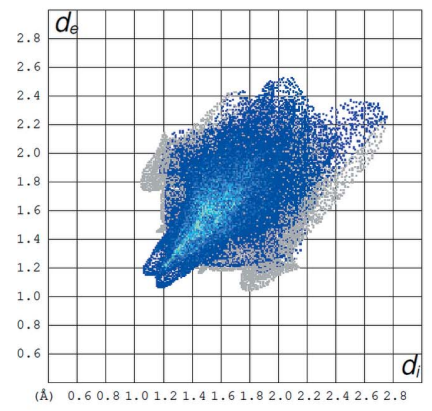

(b) $\mathrm{H}$...H

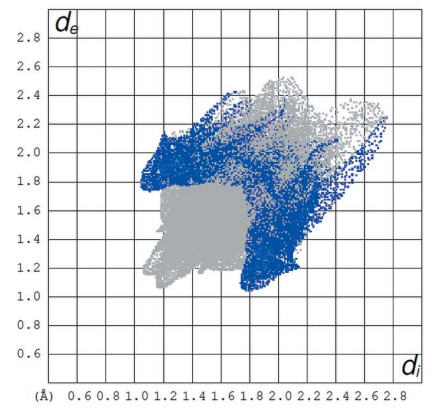

(d) $\mathbf{C} \cdots \mathbf{H} / \mathbf{H} \cdots \mathbf{C}$

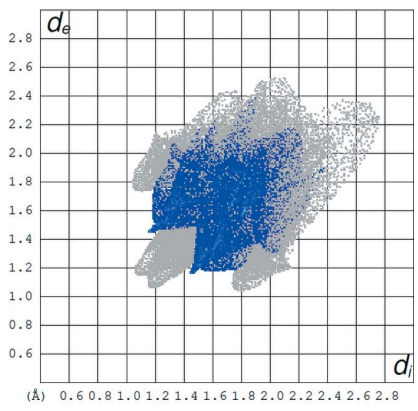

(c) $\mathbf{O} \cdots \mathbf{H} / \mathbf{H} \cdots \mathbf{O}$

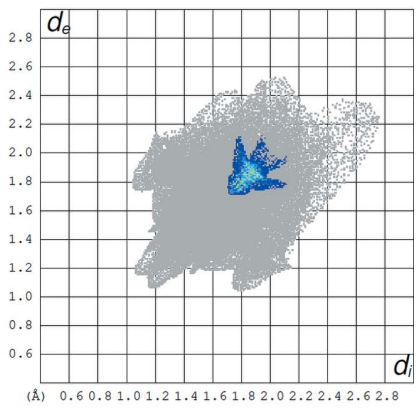

(e) $\mathrm{C} \cdots \mathrm{C}$
Figure 6

Fingerprint plots showing (a) all intermolecular interactions and delineated into $(b) \mathrm{H} \cdots \mathrm{H},(c) \mathrm{O} \cdots \mathrm{H} / \mathrm{H} \cdots \mathrm{O},(d) \mathrm{C} \cdots \mathrm{H} / \mathrm{H} \cdots \mathrm{C}$ and $(e)$ C...C contacts.

yl)ethylidene]acetohydrazide, II (TODMEH), 2-(2-nitro-1Himidazol-1-yl)- $N^{\prime}$-[1-(pyridin-2-yl)ethylidene]acetohydrazide, III (TODMIL), 2-(4-nitro- $1 H$-imidazol-1-yl)- $N^{\prime}$-[phenyl(pyridin-2-yl)methylidene]acetohydrazide, IV (TODMOR) and 2-(4-nitro-1 $H$-imidazol-1-yl)- $N^{\prime}$-[phenyl(pyridin-2-yl)methylidene]acetohydrazide, V (TODMUX). Compound I crystallizes in the monoclinic space group $C 2 / c$ with $Z=8$. In the crystal of $\mathbf{I}$, pairs of molecules are linked into dimers by $\mathrm{N}-\mathrm{H} \cdots \mathrm{O}$ hydrogen bonds, forming an $R_{2}^{2}(12)$ ring motif, with the dimers stacked along the $a$-axis direction. These dimers are connected through $\pi-\pi$ stacking interactions between the centroids of the benzene and furan rings of their 2,3-dihydro1-benzofuran ring systems. Furthermore, there exists a $\mathrm{C}-$ $\mathrm{H} \cdots \pi$ interaction that consolidates the crystal packing. Compounds II and IV crystallize in the monoclinic space group $P 2_{1} / c$ with $Z=4$. Compound III crystallizes in the monoclinic space group $I 2 / a$ with $Z=8$ and $\mathbf{V}$ crystallizes in the triclinic space group $P \overline{1}$ with $Z=2$. Compound VI crystallizes in the monoclinic space group $P 2_{1} / c$ with $Z=4$. The $E$ conformation in II, III and $\mathbf{V}$ is stabilized by a strong intermolecular $\mathrm{N}-\mathrm{H} \cdots \mathrm{O}$ interaction. These interactions lead to the formation of dimeric structural arrangements. In the crystal of $\mathbf{I V}$, an intermolecular $\mathrm{N}-\mathrm{H} \cdots \mathrm{N}$ hydrogen bond results in a helical chain structure along the $b$-axis direction. Non-classical intermolecular $\mathrm{C}-\mathrm{H} \cdots \mathrm{N}$ and $\mathrm{C}-\mathrm{H} \cdots \mathrm{O}$ interactions are also observed in the crystals of II, III, IV and V.

\section{Synthesis and crystallization}

(Z)-3-[2-(3,5-Dimethylphenyl)hydrazinylidene]benzofuran2(3H)-one was synthesized according to the reported method (Shikhaliyev et al., 2018, 2019). A $20 \mathrm{~mL}$ screw-neck vial was charged with DMSO (10 mL), (E)-2-\{[2-(3,5-dimethylphenyl)hydrazinylidene]methyl $\}$ phenol $(240 \mathrm{mg}, 1 \mathrm{mmol})$, tetramethylethylenediamine (TMEDA) (295 mg, $2.5 \mathrm{mmol}), \mathrm{CuCl}$ ( $2 \mathrm{mg}, 0.02 \mathrm{mmol})$ and $\mathrm{CCl}_{4}(20 \mathrm{mmol}, 10$ equiv). After $1-3 \mathrm{~h}$ (until TLC analysis showed complete consumption of the corresponding Schiff base), the reaction mixture was poured into a $0.01 \mathrm{M}$ solution of $\mathrm{HCl}(100 \mathrm{~mL}, \mathrm{pH} 2-3)$, and extracted with dichloromethane $(3 \times 20 \mathrm{~mL})$. The combined organic phase was washed with water $(3 \times 20 \mathrm{~mL})$, brine $(30 \mathrm{~mL})$, dried over anhydrous $\mathrm{Na}_{2} \mathrm{SO}_{4}$ and concentrated in vacuo using a rotary evaporator. The residue was purified by column chromatography on silica gel using appropriate mixtures of hexane and dichloromethane (3/1-1/1). Then the substance was refluxed in methanol for $30 \mathrm{~min}$, and left for evaporation. After three days, single crystals of the title compound suitable for X-ray analysis were obtained. Colorless solid (65\%); m.p. $475 \mathrm{~K}$. Analysis calculated for $\mathrm{C}_{16} \mathrm{H}_{14} \mathrm{~N}_{2} \mathrm{O}_{2}(M=266.30)$ : $\mathrm{C}$ 72.17, H 5.30, N 10.52; found: C 72.13, H 5.26, N 10.48\%. ${ }^{1} \mathrm{H}$ NMR $\left(300 \mathrm{MHz}, \mathrm{CDCl}_{3}\right) \delta 12.04(1 \mathrm{H}, \mathrm{NH}), 6.79-7.69(7 \mathrm{H}$, Ar), $2.37\left(6 \mathrm{H}, 2 \mathrm{CH}_{3}\right) .{ }^{13} \mathrm{C} \mathrm{NMR}\left(75 \mathrm{MHz}, \mathrm{CDCl}_{3}\right) \delta 160.47$, 157.77, 134.91, 125.14, 124.12, 121.77, 121.56, 119.86, 118.21, $114.55,108.16,106.59,16.85$ and 16.52. ESI-MS: $m / z: 267.23$ $[M+\mathrm{H}]^{+}$.

\section{Refinement details}

Crystal data, data collection and structure refinement details are summarized in Table 3 . The amine $\mathrm{H}$ atom was located in a difference-Fourier map and refined freely $[\mathrm{N} 2-\mathrm{H} 2=$ 0.93 (2) $\AA$ ]. All C-bound $\mathrm{H}$ atoms were placed at calculated positions using a riding model, with $\mathrm{C}-\mathrm{H}=0.93$ or $0.96 \AA$, and with $U_{\text {iso }}(\mathrm{H})=1.2$ or $1.5 U_{\text {eq }}(\mathrm{C})$. The methyl group with the $\mathrm{C} 16$ atom attached to the atom $\mathrm{C} 13$ is disordered over two orientations, with occupancies of 0.67 (4) and 0.33 (4). Owing to poor agreement, nine reflections $(\overline{5} 1410,7130, \overline{11} 65$, $\overline{10} 124,1110, \overline{11} 11, \overline{8} 196, \overline{8} 08$ and $\overline{10} 174)$ were omitted during the final refinement cycle. 
Table 3

Experimental details.

\begin{tabular}{ll}
\hline Crystal data & \\
Chemical formula & $\mathrm{C}_{16} \mathrm{H}_{14} \mathrm{~N}_{2} \mathrm{O}_{2}$ \\
$M_{\mathrm{r}}$ & 266.29 \\
Crystal system, space group & Monoclinic, $P 2_{1} / c$ \\
Temperature $(\mathrm{K})$ & 296 \\
$a, b, c(\AA)$ & $8.8644(4), 19.9222(8), 8.1736(3)$ \\
$\beta\left({ }^{\circ}\right)$ & $107.240(1)$ \\
$V\left(\AA^{3}\right)$ & $1378.59(10)$ \\
$Z$ & 4 \\
Radiation type & Mo $K \alpha$ \\
$\mu\left(\mathrm{mm}^{-1}\right)$ & 0.09 \\
Crystal size $(\mathrm{mm})$ & $0.40 \times 0.21 \times 0.06$ \\
& \\
Data collection & Bruker APEXII CCD \\
Diffractometer & Multi-scan $(S A D A B S ;$ Bruker, \\
Absorption correction & $2003)$ \\
& $0.684,0.746$ \\
$T_{\text {min }}, T_{\text {max }}$ & $22343,4176,2332$ \\
No. of measured, independent and & \\
$\quad$ observed $[I>2 \sigma(I)]$ reflections & 0.058 \\
$R_{\text {int }}(\text { sin } \theta / \lambda)_{\text {max }}\left(\AA^{-1}\right)$ & 0.714 \\
& \\
Refinement & \\
$R\left[F^{2}>2 \sigma\left(F^{2}\right)\right], w R\left(F^{2}\right), S$ & $0.062,0.146,1.04$ \\
No. of reflections & 4176 \\
No. of parameters & 188 \\
H-atom treatment & $\mathrm{H}$ atoms treated by a mixture of \\
& independent and constrained \\
$\Delta \rho_{\text {max }}, \Delta \rho_{\text {min }}\left(\mathrm{e} \AA^{-3}\right)$ & refinement \\
\hline
\end{tabular}

Computer programs: APEX2 (Bruker, 2003), SAINT (Bruker, 2003), SHELXT (Sheldrick, 2015a), SHELXL (Sheldrick, 2015b), ORTEP-3 for Windows (Farrugia, 2012), PLATON (Spek, 2020).

\section{Acknowledgements}

The author's contributions are as follows. Conceptualization, NQS, MA and AB; synthesis, UFA and SHM; X-ray analysis, ZA, RKA and MA; writing (review and editing of the manuscript) ZA, MA and $\mathrm{AB}$; funding acquisition, NQS, UFA, SHM and RKA; supervision, NQS, MA and AB.

\section{Funding information}

This work was performed under the support of the Science Development Foundation under the President of the Republic of Azerbaijan (grant No. EIF-BGM-4-RFTF-1/2017-21/13/4).

\section{References}

Atioğlu, Z., Akkurt, M., Askerova, U. F., Mukhtarova, S. H., Askerov, R. K. \& Mlowe, S. (2021). Acta Cryst. E77, 907-911.

Bruker (2003). APEX2, SAINT and SADABS. Bruker AXS Inc., Madison, Wisconsin.

Farrugia, L. J. (2012). J. Appl. Cryst. 45, 849-854.

Groom, C. R., Bruno, I. J., Lightfoot, M. P. \& Ward, S. C. (2016). Acta Cryst. B72, 171-179.
Gurbanov, A. V., Kuznetsov, M. L., Demukhamedova, S. D., Alieva, I. N., Godjaev, N. M., Zubkov, F. I., Mahmudov, K. T. \& Pombeiro, A. J. L. (2020a). CrystEngComm, 22, 628-633.

Gurbanov, A. V., Kuznetsov, M. L., Mahmudov, K. T., Pombeiro, A. J. L. \& Resnati, G. (2020b). Chem. Eur. J. 26, 14833-14837.

Kopylovich, M. N., Mahmudov, K. T., Mizar, A. \& Pombeiro, A. J. L. (2011). Chem. Commun. 47, 7248-7250.

Ma, Z., Gurbanov, A. V., Maharramov, A. M., Guseinov, F. I., Kopylovich, M. N., Zubkov, F. I., Mahmudov, K. T. \& Pombeiro, A. J. L. (2017a). J. Mol. Catal. A Chem. 426, 526-533.

Ma, Z., Gurbanov, A. V., Sutradhar, M., Kopylovich, M. N., Mahmudov, K. T., Maharramov, A. M., Guseinov, F. I., Zubkov, F. I. \& Pombeiro, A. J. L. (2017b). Mol. Catal. 428, 17-23.

Ma, Z., Mahmudov, K. T., Aliyeva, V. A., Gurbanov, A. V., Guedes da Silva, M. F. C. \& Pombeiro, A. J. L. (2021). Coord. Chem. Rev. 437, 213859.

Ma, Z., Mahmudov, K. T., Aliyeva, V. A., Gurbanov, A. V. \& Pombeiro, A. J. L. (2020). Coord. Chem. Rev. 423, 213482.

Maharramov, A. M., Shikhaliyev, N. Q., Suleymanova, G. T., Gurbanov, A. V., Babayeva, G. V., Mammadova, G. Z., Zubkov, F. I., Nenajdenko, V. G., Mahmudov, K. T. \& Pombeiro, A. J. L. (2018). Dyes Pigments, 159, 135-141.

Mahmoudi, G., Afkhami, F. A., Castiñeiras, A., García-Santos, I., Gurbanov, A., Zubkov, F. I., Mitoraj, M. P., Kukułka, M., Sagan, F., Szczepanik, D. W., Konyaeva, I. A. \& Safin, D. A. (2018). Inorg. Chem. 57, 4395-4408.

Mahmoudi, G., Zaręba, J. K., Gurbanov, A. V., Bauzá, A., Zubkov, F. I., Kubicki, M., Stilinović, V., Kinzhybalo, V. \& Frontera, A. (2017). Eur. J. Inorg. Chem. pp. 4763-4772.

Mahmudov, K. T., Gurbanov, A. V., Aliyeva, V. A., Resnati, G. \& Pombeiro, A. J. L. (2020). Coord. Chem. Rev. 418, 213381.

Mahmudov, K. T., Kopylovich, M. N., Haukka, M., Mahmudova, G. S., Esmaeila, E. F., Chyragov, F. M. \& Pombeiro, A. J. L. (2013). J. Mol. Struct. 1048, 108-112.

Mizar, A., Guedes da Silva, M. F. C., Kopylovich, M. N., Mukherjee, S., Mahmudov, K. T. \& Pombeiro, A. J. L. (2012). Eur. J. Inorg. Chem. pp. 2305-2313.

Oliveira, A. P. A., Ferreira, I. P., Despaigne, A. A. R., da Silva, J. G., Vieira, A. C. S., Santos, M. S., Alexandre-Moreira, M. S., Diniz, R. \& Beraldo, H. (2019). Acta Cryst. C75, 320-328.

Sheldrick, G. M. (2015a). Acta Cryst. A71, 3-8.

Sheldrick, G. M. (2015b). Acta Cryst. C71, 3-8.

Shikhaliyev, N. Q., Ahmadova, N. E., Gurbanov, A. V., Maharramov, A. M., Mammadova, G. Z., Nenajdenko, V. G., Zubkov, F. I., Mahmudov, K. T. \& Pombeiro, A. J. L. (2018). Dyes Pigments, 150, 377-381.

Shikhaliyev, N. Q., Kuznetsov, M. L., Maharramov, A. M., Gurbanov, A. V., Ahmadova, N. E., Nenajdenko, V. G., Mahmudov, K. T. \& Pombeiro, A. J. L. (2019). CrystEngComm, 21, 5032-5038.

Shixaliyev, N. Q., Gurbanov, A. V., Maharramov, A. M., Mahmudov, K. T., Kopylovich, M. N., Martins, L. M. D. R. S., Muzalevskiy, V. M., Nenajdenko, V. G. \& Pombeiro, A. J. L. (2014). New J. Chem. 38, 4807-4815.

Spek, A. L. (2020). Acta Cryst. E76, 1-11.

Turner, M. J., McKinnon, J. J., Wolff, S. K., Grimwood, D. J., Spackman, P. R., Jayatilaka, D. \& Spackman, M. A. (2017). CrystalExplorer17. The University of Western Australia.

Viswanathan, A., Kute, D., Musa, A., Konda Mani, S., Sipilä, V., Emmert-Streib, F., Zubkov, F. I., Gurbanov, A. V., Yli-Harja, O. \& Kandhavelu, M. (2019). Eur. J. Med. Chem. 166, 291-303. 


\section{supporting information}

Acta Cryst. (2021). E77, 1280-1284 [https://doi.org/10.1107/S2056989021011749]

Crystal structure and Hirshfeld surface analysis of 3-[2-(3,5-dimethylphenyl)hydrazinylidene]benzofuran-2(3H)-one

Zeliha Atioğlu, Mehmet Akkurt, Namiq Q. Shikhaliyev, Ulviyya F. Askerova, Sevinc H.

Mukhtarova, Rizvan K. Askerov and Ajaya Bhattarai

Computing details

Data collection: APEX2 (Bruker, 2003); cell refinement: SAINT (Bruker, 2003); data reduction: SAINT (Bruker, 2003); program(s) used to solve structure: SHELXT (Sheldrick, 2015a); program(s) used to refine structure: SHELXL (Sheldrick, 2015b); molecular graphics: ORTEP-3 for Windows (Farrugia, 2012); software used to prepare material for publication: PLATON (Spek, 2020).

3-[2-(3,5-Dimethylphenyl)hydrazinylidene]benzofuran-2(3H)-one

Crystal data

$\mathrm{C}_{16} \mathrm{H}_{14} \mathrm{~N}_{2} \mathrm{O}_{2}$

$M_{r}=266.29$

Monoclinic, $P 2_{1} / c$

$a=8.8644$ (4) $\AA$

$b=19.9222(8) \AA$

$c=8.1736(3) \AA$

$\beta=107.240(1)^{\circ}$

$V=1378.59(10) \AA^{3}$

$Z=4$

\section{Data collection}

Bruker APEXII CCD diffractometer

$\varphi$ and $\omega$ scans

Absorption correction: multi-scan

(SADABS; Bruker, 2003)

$T_{\text {min }}=0.684, T_{\max }=0.746$

22343 measured reflections

Refinement

Refinement on $F^{2}$

Least-squares matrix: full

$R\left[F^{2}>2 \sigma\left(F^{2}\right)\right]=0.062$

$w R\left(F^{2}\right)=0.146$

$S=1.03$

4176 reflections

188 parameters

0 restraints
$F(000)=560$

$D_{\mathrm{x}}=1.283 \mathrm{Mg} \mathrm{m}^{-3}$

Mo $K \alpha$ radiation, $\lambda=0.71073 \AA$

Cell parameters from 3738 reflections

$\theta=2.4-30.5^{\circ}$

$\mu=0.09 \mathrm{~mm}^{-1}$

$T=296 \mathrm{~K}$

Prism, colourless

$0.40 \times 0.21 \times 0.06 \mathrm{~mm}$

4176 independent reflections

2332 reflections with $I>2 \sigma(I)$

$R_{\text {int }}=0.058$

$\theta_{\text {max }}=30.5^{\circ}, \theta_{\text {min }}=2.4^{\circ}$

$h=-12 \rightarrow 12$

$k=-28 \rightarrow 28$

$l=-11 \rightarrow 11$

Hydrogen site location: mixed

$\mathrm{H}$ atoms treated by a mixture of independent and constrained refinement

$w=1 /\left[\sigma^{2}\left(F_{\mathrm{o}}^{2}\right)+(0.0465 P)^{2}+0.2174 P\right]$ where $P=\left(F_{\mathrm{o}}{ }^{2}+2 F_{\mathrm{c}}{ }^{2}\right) / 3$

$(\Delta / \sigma)_{\max }<0.001$

$\Delta \rho_{\max }=0.15 \mathrm{e} \AA^{-3}$

$\Delta \rho_{\min }=-0.14$ e $\AA^{-3}$ 


\section{Special details}

Geometry. All esds (except the esd in the dihedral angle between two 1.s. planes) are estimated using the full covariance matrix. The cell esds are taken into account individually in the estimation of esds in distances, angles and torsion angles; correlations between esds in cell parameters are only used when they are defined by crystal symmetry. An approximate (isotropic) treatment of cell esds is used for estimating esds involving l.s. planes.

Fractional atomic coordinates and isotropic or equivalent isotropic displacement parameters $\left(\AA^{2}\right)$

\begin{tabular}{|c|c|c|c|c|c|}
\hline & $x$ & $y$ & $z$ & $U_{\text {iso }} * / U_{\text {eq }}$ & Occ. $(<1)$ \\
\hline $\mathrm{C} 1$ & $0.7095(2)$ & $0.56971(8)$ & $0.9270(2)$ & $0.0519(4)$ & \\
\hline $\mathrm{C} 2$ & $0.76884(18)$ & $0.52981(8)$ & $0.8095(2)$ & $0.0482(4)$ & \\
\hline $\mathrm{C} 3$ & $0.92693(18)$ & $0.55352(8)$ & $0.8274(2)$ & $0.0493(4)$ & \\
\hline $\mathrm{C} 4$ & $1.0448(2)$ & $0.53565(9)$ & $0.7563(3)$ & $0.0624(5)$ & \\
\hline $\mathrm{H} 4$ & 1.028478 & 0.502040 & 0.673914 & $0.075^{*}$ & \\
\hline $\mathrm{C} 5$ & $1.1879(2)$ & $0.56929(11)$ & $0.8114(3)$ & $0.0728(6)$ & \\
\hline H5 & 1.268324 & 0.558312 & 0.764500 & $0.087 *$ & \\
\hline C6 & $1.2129(2)$ & $0.61884(11)$ & $0.9347(3)$ & $0.0749(6)$ & \\
\hline H6 & 1.310276 & 0.640405 & 0.969456 & $0.090 *$ & \\
\hline $\mathrm{C} 7$ & $1.0974(2)$ & $0.63715(10)$ & $1.0075(3)$ & $0.0673(5)$ & \\
\hline H7 & 1.114142 & 0.670299 & 1.091089 & $0.081 *$ & \\
\hline $\mathrm{C} 8$ & 0.95594 (19) & $0.60361(8)$ & $0.9495(2)$ & $0.0538(4)$ & \\
\hline $\mathrm{C} 9$ & $0.4768(2)$ & $0.40926(8)$ & $0.6127(2)$ & $0.0530(4)$ & \\
\hline $\mathrm{C} 10$ & $0.5476(2)$ & $0.37321(9)$ & $0.5108(2)$ & $0.0603(5)$ & \\
\hline H10 & 0.648784 & 0.384407 & 0.508401 & $0.072 *$ & \\
\hline C11 & 0.4678 (3) & $0.32049(9)$ & $0.4125(2)$ & $0.0702(6)$ & \\
\hline $\mathrm{C} 12$ & $0.3181(3)$ & $0.30497(10)$ & $0.4188(3)$ & $0.0779(6)$ & \\
\hline H12 & 0.263853 & 0.269826 & 0.351887 & $0.093^{*}$ & \\
\hline $\mathrm{C} 13$ & $0.2457(2)$ & $0.33984(10)$ & $0.5213(3)$ & $0.0693(6)$ & \\
\hline $\mathrm{C} 14$ & $0.3266(2)$ & $0.39267(9)$ & $0.6189(2)$ & $0.0598(5)$ & \\
\hline H14 & 0.280284 & 0.416993 & 0.688386 & $0.072 *$ & \\
\hline C15 & $0.5458(3)$ & $0.28029(12)$ & $0.3040(3)$ & $0.1039(9)$ & \\
\hline $\mathrm{H} 15 \mathrm{~A}$ & 0.513973 & 0.234174 & 0.302284 & $0.156^{*}$ & \\
\hline H15B & 0.658448 & 0.283384 & 0.351251 & $0.156^{*}$ & \\
\hline $\mathrm{H} 15 \mathrm{C}$ & 0.514651 & 0.297710 & 0.189347 & $0.156^{*}$ & \\
\hline C16 & $0.0820(3)$ & $0.32121(12)$ & $0.5296(3)$ & $0.0972(8)$ & \\
\hline H16D & 0.052299 & 0.350758 & 0.607547 & $0.146^{*}$ & $0.67(4)$ \\
\hline H16E & 0.082700 & 0.275746 & 0.568674 & $0.146^{*}$ & $0.67(4)$ \\
\hline $\mathrm{H} 16 \mathrm{~F}$ & 0.007489 & 0.325362 & 0.417644 & $0.146^{*}$ & $0.67(4)$ \\
\hline H16A & 0.013993 & 0.359673 & 0.500891 & $0.146^{*}$ & $0.33(4)$ \\
\hline H16B & 0.088531 & 0.306500 & 0.643291 & $0.146^{*}$ & $0.33(4)$ \\
\hline H16C & 0.039964 & 0.285692 & 0.449682 & $0.146^{*}$ & $0.33(4)$ \\
\hline N1 & $0.69683(16)$ & $0.48115(7)$ & $0.70999(18)$ & $0.0508(3)$ & \\
\hline $\mathrm{N} 2$ & $0.55439(17)$ & $0.46316(7)$ & $0.7133(2)$ & $0.0545(4)$ & \\
\hline $\mathrm{H} 2$ & $0.513(2)$ & $0.4843(10)$ & $0.792(3)$ & $0.077(6)^{*}$ & \\
\hline $\mathrm{O} 1$ & $0.82605(14)$ & $0.61480(6)$ & $1.00954(16)$ & 0.0609 (3) & \\
\hline $\mathrm{O} 2$ & $0.58433(14)$ & $0.56775(7)$ & 0.95705 (17) & $0.0666(4)$ & \\
\hline
\end{tabular}


Atomic displacement parameters $\left(\AA^{2}\right)$

\begin{tabular}{lllllll}
\hline & $U^{11}$ & $U^{22}$ & $U^{33}$ & $U^{12}$ & $U^{13}$ & $U^{23}$ \\
\hline C1 & $0.0428(9)$ & $0.0531(9)$ & $0.0561(10)$ & $-0.0041(7)$ & $0.0089(8)$ & $-0.0012(8)$ \\
C2 & $0.0422(9)$ & $0.0501(9)$ & $0.0482(9)$ & $-0.0004(7)$ & $0.0073(7)$ & $0.0016(7)$ \\
C3 & $0.0403(8)$ & $0.0503(9)$ & $0.0546(10)$ & $0.0026(7)$ & $0.0100(7)$ & $0.0083(7)$ \\
C4 & $0.0525(11)$ & $0.0673(11)$ & $0.0680(12)$ & $0.0056(9)$ & $0.0186(9)$ & $0.0090(9)$ \\
C5 & $0.0459(11)$ & $0.0842(14)$ & $0.0898(16)$ & $0.0066(10)$ & $0.0223(11)$ & $0.0224(12)$ \\
C6 & $0.0445(11)$ & $0.0770(13)$ & $0.0958(16)$ & $-0.0094(9)$ & $0.0092(11)$ & $0.0179(12)$ \\
C7 & $0.0512(11)$ & $0.0607(11)$ & $0.0808(14)$ & $-0.0092(9)$ & $0.0053(10)$ & $0.0015(10)$ \\
C8 & $0.0409(9)$ & $0.0531(9)$ & $0.0630(11)$ & $-0.0004(7)$ & $0.0088(8)$ & $0.0055(8)$ \\
C9 & $0.0536(10)$ & $0.0501(9)$ & $0.0461(9)$ & $-0.0056(8)$ & $0.0005(8)$ & $0.0030(7)$ \\
C10 & $0.0700(12)$ & $0.0553(10)$ & $0.0511(10)$ & $-0.0063(9)$ & $0.0109(9)$ & $0.0003(8)$ \\
C11 & $0.0950(16)$ & $0.0537(11)$ & $0.0542(11)$ & $-0.0090(10)$ & $0.0102(11)$ & $-0.0011(9)$ \\
C12 & $0.0988(17)$ & $0.0575(12)$ & $0.0572(12)$ & $-0.0205(11)$ & $-0.0078(12)$ & $-0.0005(10)$ \\
C13 & $0.0649(12)$ & $0.0635(12)$ & $0.0621(12)$ & $-0.0153(9)$ & $-0.0080(10)$ & $0.0156(10)$ \\
C14 & $0.0534(10)$ & $0.0616(11)$ & $0.0553(10)$ & $-0.0066(8)$ & $0.0020(8)$ & $0.0065(8)$ \\
C15 & $0.150(2)$ & $0.0746(15)$ & $0.0865(17)$ & $-0.0083(15)$ & $0.0348(17)$ & $-0.0243(13)$ \\
C16 & $0.0724(15)$ & $0.0949(17)$ & $0.1019(18)$ & $-0.0327(12)$ & $-0.0085(13)$ & $0.0192(14)$ \\
N1 & $0.0466(8)$ & $0.0525(8)$ & $0.0497(8)$ & $-0.0017(6)$ & $0.0086(6)$ & $0.0028(6)$ \\
N2 & $0.0475(8)$ & $0.0574(9)$ & $0.0553(9)$ & $-0.0059(7)$ & $0.0100(7)$ & $-0.0065(7)$ \\
O1 & $0.0496(7)$ & $0.0610(7)$ & $0.0690(8)$ & $-0.0061(6)$ & $0.0127(6)$ & $-0.0130(6)$ \\
O2 & $0.0473(7)$ & $0.0790(9)$ & $0.0757(9)$ & $-0.0041(6)$ & $0.0213(6)$ & $-0.0101(7)$ \\
& & & & & & \\
\hline
\end{tabular}

Geometric parameters $\left(A,{ }^{\circ}\right)$

\begin{tabular}{llll}
\hline $\mathrm{C} 1-\mathrm{O} 2$ & $1.2064(19)$ & $\mathrm{C} 10-\mathrm{H} 10$ & 0.9300 \\
$\mathrm{C} 1-\mathrm{O} 1$ & $1.3842(19)$ & $\mathrm{C} 11-\mathrm{C} 12$ & $1.378(3)$ \\
$\mathrm{C} 1-\mathrm{C} 2$ & $1.459(2)$ & $\mathrm{C} 11-\mathrm{C} 15$ & $1.506(3)$ \\
$\mathrm{C} 2-\mathrm{N} 1$ & $1.304(2)$ & $\mathrm{C} 12-\mathrm{C} 13$ & $1.384(3)$ \\
$\mathrm{C} 2-\mathrm{C} 3$ & $1.445(2)$ & $\mathrm{C} 12-\mathrm{H} 12$ & 0.9300 \\
$\mathrm{C} 3-\mathrm{C} 8$ & $1.380(2)$ & $\mathrm{C} 13-\mathrm{C} 14$ & $1.385(2)$ \\
$\mathrm{C} 3-\mathrm{C} 4$ & $1.385(2)$ & $\mathrm{C} 13-\mathrm{C} 16$ & $1.519(3)$ \\
$\mathrm{C} 4-\mathrm{C} 5$ & $1.386(3)$ & $\mathrm{C} 14-\mathrm{H} 14$ & 0.9300 \\
$\mathrm{C} 4-\mathrm{H} 4$ & 0.9300 & $\mathrm{C} 15-\mathrm{H} 15 \mathrm{~A}$ & 0.9600 \\
$\mathrm{C} 5-\mathrm{C} 6$ & $1.381(3)$ & $\mathrm{C} 15-\mathrm{H} 15 \mathrm{~B}$ & 0.9600 \\
$\mathrm{C} 5-\mathrm{H} 5$ & 0.9300 & $\mathrm{C} 15-\mathrm{H} 15 \mathrm{C}$ & 0.9600 \\
$\mathrm{C} 6-\mathrm{C} 7$ & $1.377(3)$ & $\mathrm{C} 16-\mathrm{H} 16 \mathrm{D}$ & 0.9600 \\
$\mathrm{C} 6-\mathrm{H} 6$ & 0.9300 & $\mathrm{C} 16-\mathrm{H} 16 \mathrm{E}$ & 0.9600 \\
$\mathrm{C} 7-\mathrm{C} 8$ & $1.375(2)$ & $\mathrm{C} 16-\mathrm{H} 16 \mathrm{~F}$ & 0.9600 \\
$\mathrm{C} 7-\mathrm{H} 7$ & 0.9300 & $\mathrm{C} 16-\mathrm{H} 16 \mathrm{~A}$ & 0.9600 \\
$\mathrm{C} 8-\mathrm{O} 1$ & $1.397(2)$ & $\mathrm{C} 16-\mathrm{H} 16 \mathrm{~B}$ & 0.9600 \\
$\mathrm{C} 9-\mathrm{C} 10$ & $1.383(2)$ & $\mathrm{C} 16-\mathrm{H} 16 \mathrm{C}$ & 0.9600 \\
$\mathrm{C} 9-\mathrm{C} 14$ & $1.387(2)$ & $\mathrm{N} 1-\mathrm{N} 2$ & $1.3206(19)$ \\
$\mathrm{C} 9-\mathrm{N} 2$ & $1.402(2)$ & $\mathrm{N} 2-\mathrm{H} 2$ & $0.93(2)$ \\
$\mathrm{C} 10-\mathrm{C} 11$ & & \\
$\mathrm{O} 2-\mathrm{C} 1-\mathrm{O} 1$ & $1.383(2)$ & $\mathrm{C} 11-\mathrm{C} 12-\mathrm{C} 13$ & $122.29(18)$
\end{tabular}




\begin{tabular}{|c|c|c|c|}
\hline $\mathrm{O} 2-\mathrm{C} 1-\mathrm{C} 2$ & $130.48(16)$ & $\mathrm{C} 11-\mathrm{C} 12-\mathrm{H} 12$ & 118.9 \\
\hline $\mathrm{O} 1-\mathrm{C} 1-\mathrm{C} 2$ & $108.18(14)$ & $\mathrm{C} 13-\mathrm{C} 12-\mathrm{H} 12$ & 118.9 \\
\hline $\mathrm{N} 1-\mathrm{C} 2-\mathrm{C} 3$ & $125.97(16)$ & $\mathrm{C} 12-\mathrm{C} 13-\mathrm{C} 14$ & $118.4(2)$ \\
\hline $\mathrm{N} 1-\mathrm{C} 2-\mathrm{C} 1$ & $127.59(15)$ & $\mathrm{C} 12-\mathrm{C} 13-\mathrm{C} 16$ & $121.7(2)$ \\
\hline $\mathrm{C} 3-\mathrm{C} 2-\mathrm{C} 1$ & $106.43(14)$ & $\mathrm{C} 14-\mathrm{C} 13-\mathrm{C} 16$ & $119.9(2)$ \\
\hline $\mathrm{C} 8-\mathrm{C} 3-\mathrm{C} 4$ & $119.17(16)$ & $\mathrm{C} 13-\mathrm{C} 14-\mathrm{C} 9$ & $119.93(19)$ \\
\hline $\mathrm{C} 8-\mathrm{C} 3-\mathrm{C} 2$ & $106.10(15)$ & $\mathrm{C} 13-\mathrm{C} 14-\mathrm{H} 14$ & 120.0 \\
\hline $\mathrm{C} 4-\mathrm{C} 3-\mathrm{C} 2$ & $134.70(17)$ & C9-C14-H14 & 120.0 \\
\hline $\mathrm{C} 3-\mathrm{C} 4-\mathrm{C} 5$ & $118.10(19)$ & $\mathrm{C} 11-\mathrm{C} 15-\mathrm{H} 15 \mathrm{~A}$ & 109.5 \\
\hline $\mathrm{C} 3-\mathrm{C} 4-\mathrm{H} 4$ & 121.0 & $\mathrm{C} 11-\mathrm{C} 15-\mathrm{H} 15 \mathrm{~B}$ & 109.5 \\
\hline $\mathrm{C} 5-\mathrm{C} 4-\mathrm{H} 4$ & 121.0 & $\mathrm{H} 15 \mathrm{~A}-\mathrm{C} 15-\mathrm{H} 15 \mathrm{~B}$ & 109.5 \\
\hline $\mathrm{C} 6-\mathrm{C} 5-\mathrm{C} 4$ & 121.07 (19) & $\mathrm{C} 11-\mathrm{C} 15-\mathrm{H} 15 \mathrm{C}$ & 109.5 \\
\hline $\mathrm{C} 6-\mathrm{C} 5-\mathrm{H} 5$ & 119.5 & $\mathrm{H} 15 \mathrm{~A}-\mathrm{C} 15-\mathrm{H} 15 \mathrm{C}$ & 109.5 \\
\hline $\mathrm{C} 4-\mathrm{C} 5-\mathrm{H} 5$ & 119.5 & $\mathrm{H} 15 \mathrm{~B}-\mathrm{C} 15-\mathrm{H} 15 \mathrm{C}$ & 109.5 \\
\hline $\mathrm{C} 7-\mathrm{C} 6-\mathrm{C} 5$ & $121.71(19)$ & $\mathrm{C} 13-\mathrm{C} 16-\mathrm{H} 16 \mathrm{D}$ & 109.5 \\
\hline $\mathrm{C} 7-\mathrm{C} 6-\mathrm{H} 6$ & 119.1 & $\mathrm{C} 13-\mathrm{C} 16-\mathrm{H} 16 \mathrm{E}$ & 109.5 \\
\hline $\mathrm{C} 5-\mathrm{C} 6-\mathrm{H} 6$ & 119.1 & $\mathrm{H} 16 \mathrm{D}-\mathrm{C} 16-\mathrm{H} 16 \mathrm{E}$ & 109.5 \\
\hline $\mathrm{C} 8-\mathrm{C} 7-\mathrm{C} 6$ & 116.17 (19) & $\mathrm{C} 13-\mathrm{C} 16-\mathrm{H} 16 \mathrm{~F}$ & 109.5 \\
\hline $\mathrm{C} 8-\mathrm{C} 7-\mathrm{H} 7$ & 121.9 & $\mathrm{H} 16 \mathrm{D}-\mathrm{C} 16-\mathrm{H} 16 \mathrm{~F}$ & 109.5 \\
\hline $\mathrm{C} 6-\mathrm{C} 7-\mathrm{H} 7$ & 121.9 & $\mathrm{H} 16 \mathrm{E}-\mathrm{C} 16-\mathrm{H} 16 \mathrm{~F}$ & 109.5 \\
\hline $\mathrm{C} 7-\mathrm{C} 8-\mathrm{C} 3$ & $123.77(18)$ & $\mathrm{C} 13-\mathrm{C} 16-\mathrm{H} 16 \mathrm{~A}$ & 109.5 \\
\hline $\mathrm{C} 7-\mathrm{C} 8-\mathrm{O} 1$ & $124.32(17)$ & $\mathrm{C} 13-\mathrm{C} 16-\mathrm{H} 16 \mathrm{~B}$ & 109.5 \\
\hline $\mathrm{C} 3-\mathrm{C} 8-\mathrm{O} 1$ & $111.89(14)$ & $\mathrm{H} 16 \mathrm{~A}-\mathrm{C} 16-\mathrm{H} 16 \mathrm{~B}$ & 109.5 \\
\hline $\mathrm{C} 10-\mathrm{C} 9-\mathrm{C} 14$ & $120.63(16)$ & $\mathrm{C} 13-\mathrm{C} 16-\mathrm{H} 16 \mathrm{C}$ & 109.5 \\
\hline $\mathrm{C} 10-\mathrm{C} 9-\mathrm{N} 2$ & $121.31(16)$ & $\mathrm{H} 16 \mathrm{~A}-\mathrm{C} 16-\mathrm{H} 16 \mathrm{C}$ & 109.5 \\
\hline $\mathrm{C} 14-\mathrm{C} 9-\mathrm{N} 2$ & $118.05(17)$ & $\mathrm{H} 16 \mathrm{~B}-\mathrm{C} 16-\mathrm{H} 16 \mathrm{C}$ & 109.5 \\
\hline $\mathrm{C} 11-\mathrm{C} 10-\mathrm{C} 9$ & $119.94(19)$ & $\mathrm{C} 2-\mathrm{N} 1-\mathrm{N} 2$ & $118.89(15)$ \\
\hline $\mathrm{C} 11-\mathrm{C} 10-\mathrm{H} 10$ & 120.0 & $\mathrm{~N} 1-\mathrm{N} 2-\mathrm{C} 9$ & $120.08(15)$ \\
\hline $\mathrm{C} 9-\mathrm{C} 10-\mathrm{H} 10$ & 120.0 & $\mathrm{~N} 1-\mathrm{N} 2-\mathrm{H} 2$ & $117.9(13)$ \\
\hline $\mathrm{C} 12-\mathrm{C} 11-\mathrm{C} 10$ & $118.8(2)$ & $\mathrm{C} 9-\mathrm{N} 2-\mathrm{H} 2$ & $121.7(13)$ \\
\hline $\mathrm{C} 12-\mathrm{C} 11-\mathrm{C} 15$ & $121.18(19)$ & $\mathrm{C} 1-\mathrm{O} 1-\mathrm{C} 8$ & $107.39(13)$ \\
\hline $\mathrm{C} 10-\mathrm{C} 11-\mathrm{C} 15$ & $120.0(2)$ & & \\
\hline $\mathrm{O} 2-\mathrm{C} 1-\mathrm{C} 2-\mathrm{N} 1$ & $-1.0(3)$ & $\mathrm{N} 2-\mathrm{C} 9-\mathrm{C} 10-\mathrm{C} 11$ & $-179.74(16)$ \\
\hline $\mathrm{O} 1-\mathrm{C} 1-\mathrm{C} 2-\mathrm{N} 1$ & $178.73(15)$ & $\mathrm{C} 9-\mathrm{C} 10-\mathrm{C} 11-\mathrm{C} 12$ & $-0.1(3)$ \\
\hline $\mathrm{O} 2-\mathrm{C} 1-\mathrm{C} 2-\mathrm{C} 3$ & $-179.52(18)$ & $\mathrm{C} 9-\mathrm{C} 10-\mathrm{C} 11-\mathrm{C} 15$ & $-178.83(18)$ \\
\hline $\mathrm{O} 1-\mathrm{C} 1-\mathrm{C} 2-\mathrm{C} 3$ & $0.23(17)$ & $\mathrm{C} 10-\mathrm{C} 11-\mathrm{C} 12-\mathrm{C} 13$ & $-0.7(3)$ \\
\hline $\mathrm{N} 1-\mathrm{C} 2-\mathrm{C} 3-\mathrm{C} 8$ & $-178.02(16)$ & $\mathrm{C} 15-\mathrm{C} 11-\mathrm{C} 12-\mathrm{C} 13$ & $178.06(19)$ \\
\hline $\mathrm{C} 1-\mathrm{C} 2-\mathrm{C} 3-\mathrm{C} 8$ & $0.52(17)$ & $\mathrm{C} 11-\mathrm{C} 12-\mathrm{C} 13-\mathrm{C} 14$ & $0.8(3)$ \\
\hline $\mathrm{N} 1-\mathrm{C} 2-\mathrm{C} 3-\mathrm{C} 4$ & $-0.4(3)$ & $\mathrm{C} 11-\mathrm{C} 12-\mathrm{C} 13-\mathrm{C} 16$ & $-178.75(19)$ \\
\hline $\mathrm{C} 1-\mathrm{C} 2-\mathrm{C} 3-\mathrm{C} 4$ & $178.17(19)$ & $\mathrm{C} 12-\mathrm{C} 13-\mathrm{C} 14-\mathrm{C} 9$ & $-0.3(3)$ \\
\hline $\mathrm{C} 8-\mathrm{C} 3-\mathrm{C} 4-\mathrm{C} 5$ & $-0.1(3)$ & $\mathrm{C} 16-\mathrm{C} 13-\mathrm{C} 14-\mathrm{C} 9$ & $179.33(17)$ \\
\hline $\mathrm{C} 2-\mathrm{C} 3-\mathrm{C} 4-\mathrm{C} 5$ & $-177.49(18)$ & $\mathrm{C} 10-\mathrm{C} 9-\mathrm{C} 14-\mathrm{C} 13$ & $-0.5(3)$ \\
\hline $\mathrm{C} 3-\mathrm{C} 4-\mathrm{C} 5-\mathrm{C} 6$ & $0.5(3)$ & $\mathrm{N} 2-\mathrm{C} 9-\mathrm{C} 14-\mathrm{C} 13$ & $179.91(15)$ \\
\hline $\mathrm{C} 4-\mathrm{C} 5-\mathrm{C} 6-\mathrm{C} 7$ & $-0.3(3)$ & $\mathrm{C} 3-\mathrm{C} 2-\mathrm{N} 1-\mathrm{N} 2$ & $176.94(15)$ \\
\hline $\mathrm{C} 5-\mathrm{C} 6-\mathrm{C} 7-\mathrm{C} 8$ & $-0.4(3)$ & $\mathrm{C} 1-\mathrm{C} 2-\mathrm{N} 1-\mathrm{N} 2$ & $-1.3(2)$ \\
\hline $\mathrm{C} 6-\mathrm{C} 7-\mathrm{C} 8-\mathrm{C} 3$ & $0.9(3)$ & $\mathrm{C} 2-\mathrm{N} 1-\mathrm{N} 2-\mathrm{C} 9$ & $-177.48(14)$ \\
\hline $\mathrm{C} 6-\mathrm{C} 7-\mathrm{C} 8-\mathrm{O} 1$ & $179.25(16)$ & $\mathrm{C} 10-\mathrm{C} 9-\mathrm{N} 2-\mathrm{N} 1$ & $1.1(2)$ \\
\hline
\end{tabular}




$\begin{array}{llll}\mathrm{C} 4-\mathrm{C} 3-\mathrm{C} 8-\mathrm{C} 7 & -0.7(3) & \mathrm{C} 14-\mathrm{C} 9-\mathrm{N} 2-\mathrm{N} 1 & -179.26(15) \\ \mathrm{C} 2-\mathrm{C} 3-\mathrm{C} 8-\mathrm{C} 7 & 177.41(16) & \mathrm{O} 2-\mathrm{C} 1-\mathrm{O} 1-\mathrm{C} 8 & 178.89(16) \\ \mathrm{C} 4-\mathrm{C} 3-\mathrm{C} 8-\mathrm{O} 1 & -179.19(14) & \mathrm{C} 2-\mathrm{C} 1-\mathrm{O} 1-\mathrm{C} 8 & -0.88(17) \\ \mathrm{C} 2-\mathrm{C} 3-\mathrm{C} 8-\mathrm{O} 1 & -1.11(19) & \mathrm{C} 7-\mathrm{C} 8-\mathrm{O} 1-\mathrm{C} 1 & -177.24(17) \\ \mathrm{C} 14-\mathrm{C} 9-\mathrm{C} 10-\mathrm{C} 11 & 0.6(3) & \mathrm{C} 3-\mathrm{C} 8-\mathrm{O} 1-\mathrm{C} 1 & 1.27(19)\end{array}$

Hydrogen-bond geometry $\left(A,{ }^{\circ}\right)$

$C g 2$ and $C g 3$ are the centroids of the $\mathrm{C} 3-\mathrm{C} 8$ and $\mathrm{C} 9-\mathrm{C} 14$ rings, respectively.

\begin{tabular}{lllll}
\hline$D-\mathrm{H} \cdots A$ & $D-\mathrm{H}$ & $\mathrm{H} \cdots A$ & $D \cdots A$ & $D-\mathrm{H}^{\cdots} A$ \\
\hline $\mathrm{N} 2-\mathrm{H} 2 \cdots \mathrm{O} 2$ & $0.93(2)$ & $2.12(2)$ & $2.840(2)$ & $133.8(16)$ \\
$\mathrm{C} 15-\mathrm{H} 15 A \cdots C g 3^{\mathrm{i}}$ & 0.96 & 2.90 & $3.591(3)$ & 130 \\
$\mathrm{C} 16-\mathrm{H} 16 F \cdots C g 2^{\mathrm{ii}}$ & 0.96 & 2.92 & $3.715(3)$ & 141 \\
\hline
\end{tabular}

Symmetry codes: (i) $x,-y-1 / 2, z-3 / 2$; (ii) $-x+1,-y+1,-z+1$. 